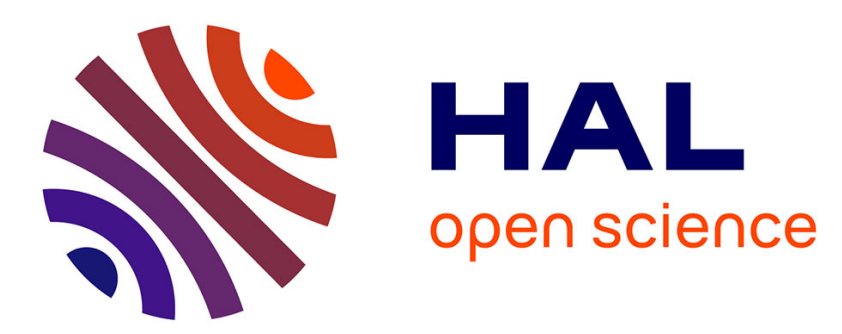

\title{
Impact of topology on plant functioning : a theoretical analysis based on the GreenLab model equations
}

Véronique Letort, Paul-Henry Cournède, Philippe de Reffye

\section{To cite this version:}

Véronique Letort, Paul-Henry Cournède, Philippe de Reffye. Impact of topology on plant functioning: a theoretical analysis based on the GreenLab model equations. 2009 Plant Growth Modeling And Applications, Nov 2009, Beijing, China. pp.341-348. hal-00546801

\section{HAL Id: hal-00546801 https://hal.science/hal-00546801}

Submitted on 15 Dec 2010

HAL is a multi-disciplinary open access archive for the deposit and dissemination of scientific research documents, whether they are published or not. The documents may come from teaching and research institutions in France or abroad, or from public or private research centers.
L'archive ouverte pluridisciplinaire HAL, est destinée au dépôt et à la diffusion de documents scientifiques de niveau recherche, publiés ou non, émanant des établissements d'enseignement et de recherche français ou étrangers, des laboratoires publics ou privés. 


\section{Impact of topology on plant functioning : a theoretical analysis based on the GreenLab model equations.}

\author{
Véronique LETORT \\ Applied Mathematics and Systems \\ Ecole Centrale Paris \\ F-92295 Châtenay-Malabry, France \\ letort@mas.ecp.fr
}

\author{
Paul-Henry COURNEDE \\ Applied Mathematics and Systems \\ Ecole Centrale Paris \\ F-92295 Châtenay-Malabry, France \\ cournede@mas.ecp.fr
}

\author{
Philippe DE REFFYE \\ AMAP/Digiplante \\ CIRAD, F-34398 Montpellier \\ \&INRIA, F-91893 Saclay, France \\ dereffye@cirad.fr
}

\begin{abstract}
A growing interest emerges for the application of plant functional-structural models to study the influence of topological development on plant growth. This kind of study is classically done through virtual experiments on simulated plants to analyze the effects of architectural changes on the model outputs. In this paper, we take advantage of the mathematical formalism developped for describing plant structure and growth in the GreenLab model to perform a theoretical analysis of the importance of topological development for plant functioning. Using the basic formulation of the model, it is possible to solve analytically the equation giving the limit biomass production under stable environment and to estimate the sensitivity of this limit value to the parameters of topological development. Reciprocally, we analyze the conditions under which plants with different architectures can have the same trajectory of biomass production in models based on Beer-Lambert-like formulations.
\end{abstract}

\section{Introduction}

It is a current scientific challenge to understand and analyze the potentialities of the different architectural models of plant structure [1]. The ongoing development of functionalstructural tree models (FSTM) [2], [3] opens new ways to study plant growth and more particularly to investigate the complex interactions between tree structure and physiological processes. Most of them represent the tree topology or geometry as a set of basic units, phytomers [4].

These models are useful tools to integrate the current biological knowledge at different scales, even for organisms as complex as trees (see for instance [5], [6]). Virtual experiments can be performed to investigate the effects of changes in plant architecture on different phenomena (e.g. simulations of horticultural strategies, such as pruning fruit trees [7], [8], interception of solar radiation [9][11], plant-pathogens interactions $[12], \ldots)$. However these models are often formulated as complicated flowcharts with a high number of parameters, including many processes and interactions. On the other hand, models such as the GreenLab model [13], [14] consider only simplified versions of biological processes so that they can be formulated in the form of dynamic systems.

Although writing a model under a mathematical form is sometimes done at the expense of its biological relevance (some phenomena are neglected), it offers some possibilities compared to mere simulation black boxes. In his review of structure modelling, Prusinkiewicz [15] presents the advantages of representing the topological development of plants using a language, and more particularly L-systems, to reduce the programming effort, to describe the plant structure in a compact and precise manner and to facilitate model comparisons. A further step is to exploit the properties that naturally stem from the use of L-systems rules, such as structural factorization [14], computation of moments of numbers of organs [16] and generating functions [17]. Kurth [18] presents additional arguments in favour of the use of an explicit formalism to describe plant architectures. Equations are more elegant and condensed than a complicated algorithm or flowchart that must be analyzed to understand the model. The model gets a higher level of transparency and of universality when an exact specification language, designed for that purpose, is used. It allows applying general theories that have been previously developed in other disciplines.

In this approach, there is a search for simplicity and for extracting the minimal set of variables necessary to simulate the growth of plants at the organ scale. In GreenLab, the minimal set of processes that has been identified represents the plant development, biomass production and biomass allocation to each organ. The associated variables are linked through recurrence equations. In the current versions of the model, biomass production is computed according to the active blade surface through an equation of the form of the Beer-Lambert law. In this study, we take advantage of this formalism to study theoretically the influence of topology on biomass production in GreenLab. In particular, we show that the biomass production stabilizes as time goes to infinity and we determine the conditions under which this limit value is non-zero. We define an index based on 
the topological parameters of the model to evaluate the potentialities of different architectural models to reach high values of limit biomass production. We show that under particular conditions, plants with different topological developments can have exactly the same trajectory of biomass production over time. This last property helps bridging the gap between process-based models and functional-structural models, as it shows that under the appropriate assumptions, the topological development has no influence on the plant prodution.

\section{Limit production of biomass according to its topological model}

The limit behaviour of a model is defined as the limit of its state variables when times tends to infinity with constant input parameters and under stable control variables. In this paper, we focus more particularly on the limit biomass production when the number of growth cycles increases to infinity.

\subsection{The growth equation of the GreenLab model}

Botanical analyses of tree architecture have shown that trees exhibit a hierarchical organization and repetitive sequences of the same structures [19]. Therefore, axes in a plant can be sorted according to their morphological or functional propoperties and indexed thanks to the notion of physiological age of the apical meristem. In the GreenLab model, plant development is simulated using a dual-scale automaton [20] whose states and transition rules are based on that index of physiological age. As shown in [?], [16], this method is analogous to writing a growth grammar, as done in simulations based on L-Systems [21]. The rules defining the plant development can be either deterministic [13], stochastic [22] or mechanistic [23]. The alphabet of GreenLab is introduced in [14], [24]: it is used to recursively describe tree structure (concept of structural factorization) and to define morphisms representing operations on the plant, e.g. the growth morphism. Another interesting application is the possibility to analytically compute the number of organs appearing at each growth cycle in the deterministic and mechanistic versions of GreenLab [14] and the moments or generating functions of numbers of organs in the stochastic case [?], [16].

As is the case for development, the evolution of the physiological variables can be expressed using dynamics equations. As the model considered here is based on discrete time steps (called growth cycles), the computation of biomass production at a given cycle can be explicitly written according to the state variables of the model at the previous cycles.

Assuming that the resistivities of internodes and petioles are negligible, the biomass production is supposed to only depend on the plant total blade area. The plant development influences the value of $S(t)$, as blades have to compete with other organs to get their biomass. Detailed presentations of the GreenLab model can be found in [13], [14], [23], [25]. We present here the generic recurrence equation of GreenLab, linking the production of biomass $Q(t)$ at cycle $t$ to the state variables of the system at the previous growth cycles:

$$
\begin{aligned}
& \forall t \geq t_{a}, Q(t)=E(t) \mu S_{p} \\
& {\left[1-\exp \left(\frac{-k}{e S_{p}} \sum_{i=1}^{t_{a}} N_{t-i+1}^{a} \sum_{j=1}^{i} \frac{\phi_{j}^{a} Q(t-i+j-1)}{D(t-i+j-1)}\right)\right]}
\end{aligned}
$$

where $E(t)$ is the environmental factor during cycle $t, e$ denotes specific blade mass, $\mu$ is a parameter of biomass conversion efficiency, $k$ is the Beer coefficient of light extinction, $S_{p}$ is a characteristic surface related to the ground projection of the plant, $t^{a}$ is the duration of blade photosynthetic activity and $N^{a}(t-i+1)$ is the number of leaves appearing at cycle $t-i+1$. Blade sinks are defined by their sink variation functions $\phi^{a}(j)$. For the sake of clarity, we present here the case when blades of every physiological age have the same sink strength, equal to 1 . Note that if different sink values $P_{k}^{a}$ for blades have to be taken into account, the term $N^{a}(t-i+1)$ simply needs to be replaced by $\sum_{k=1}^{P_{m}} N_{k}^{a}(t-i+1) P_{k}^{a}, P_{m}$ being the maximal index of physiological age in the plant and $N_{k}^{a}(t-i+1)$ the number of blades of physiological age $k$ appeared at cycle $t-i+1$. The domain of definition of the sink variation function of every organ $o, \phi^{o}: j \mapsto \phi^{o}(j)$, is $\left\{0, \ldots, t_{a}\right\}$ although the expansion duration $t_{\text {exp }}$ can be shorter than its time of activity. The function takes the value 0 in the interval $\left\{t_{\text {exp }}, \ldots, t_{a}\right\}$.

This expression shows that the plant development influences the production through the demand (that includes numbers of organs appearing at each growth cycle) and the numbers of blades.

The limit production of the model is defined as the production of the plant when the number of growth cycles $t$ goes to infinity. We investigate under which conditions the plant production can reach a stable non-zero phase. Of course the notion of infinite number of growth cycles is purely theoretical but in practice the stable regime can be reached after a phase of growth establishment. In this stable regime, the plant production is equal (or tends to) the limit production. If the limit production is zero, it means that the simulated plant does not survive. Therefore it is interesting to compare the performances of different architectural models. 


\subsection{Calculation of limit biomass production}

Due to the exponential term, the production is always bounded:

$$
\forall t, Q(t) \leq E(t) \mu S_{p}
$$

Let us first consider the case of a plant with a fixed deterministic development $(G L 1)$ with no or negligible ring compartment growing under constant environmental conditions or at least under conditions that tend to stabilize $\left(\lim _{t \rightarrow \infty} E(t)=E_{\infty}\right)$. Thus the notion of topological development only refers to the number of organs appearing at each cycle. According to the fixed point theorem, the iterated function sequence, if it converges, tends to the solution of the equation:

$$
x=A \cdot\left(1-e^{-B \cdot x}\right)
$$

where:

$$
\begin{aligned}
& A=E_{\infty} \mu S_{p} \\
& B=\frac{-k}{e S_{p}} \sum_{i=1}^{t_{a}} \sum_{j=1}^{i} \phi^{a}(j)\left(\frac{N^{a}}{D}\right)_{\infty}
\end{aligned}
$$

with $\left(\frac{N^{a}}{D}\right)_{\infty}=\lim _{t \rightarrow \infty} \frac{N^{a}(t)}{D(t)}$. Its value depends on the plant development. It can be proved that this limit exists in the deterministic case [26], [27] but in the mechanistic case (GL3), there can be emergence of periodic oscillations of this ratio [28] so only majorations of plant production can be written. Hereafter we consider that $B$ converges and reaches its limit.

The function $f: \mathbb{R} \rightarrow \mathbb{R}$ as: $f(x)=A\left(1-e^{-B x}\right)$ admits a non zero solution if and only if $f^{\prime}(0)>1$, i.e. $A B>1$. Therefore, the condition to reach a positive limit value of biomass production is:

$$
\frac{E_{\infty} \mu k}{e} \sum_{i=1}^{t_{a}} \sum_{j=1}^{i} \phi^{a}(j)\left(\frac{N^{a}}{D}\right)_{\infty}>1
$$

Corless et al [29] (see also [30]) has proved that in that case, the solution of this equation is:

$$
Q_{\infty}=A+\frac{1}{B} \cdot W\left(-A B \cdot e^{-A B}\right)
$$

where $W$ is the Lambert function. The Lambert function, also called the omega function, is the inverse function of $h$ defined by $h(w)=w e^{w}$. It means that $W(x)$ is solution of the equation:

$$
W(x) e^{W(x)}=x
$$

As $h$ is non injective, $W$ is multivalued: on the interval $\left[-\frac{1}{e} ; 0\right), W(x)$ takes two values, as shown in Figure 1.

As $A B>1 \Rightarrow-A B \cdot e^{-A B} \in\left(-\frac{1}{e} ; 0\right)$, there are two possible values for $W$ in equation 4 . One of these solutions is $W(x)=-A B$, wich is lower than -1 and gives the

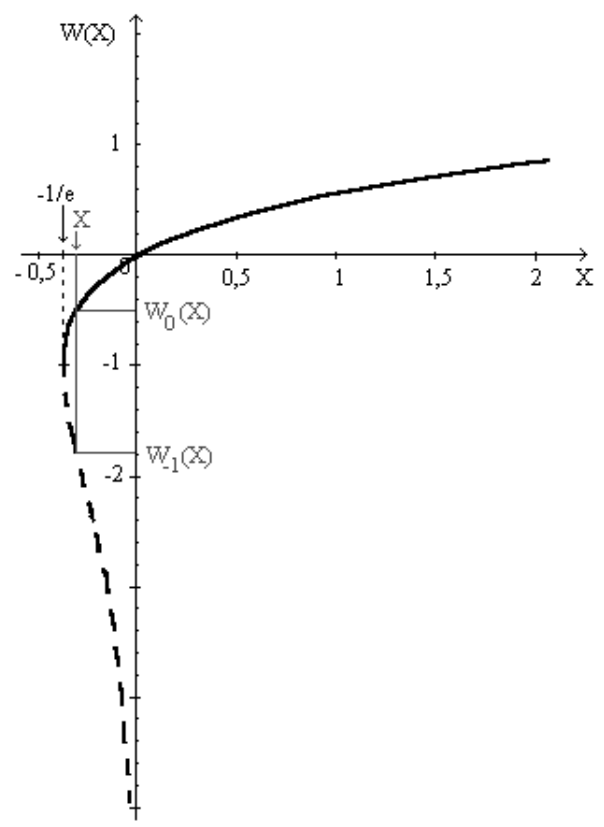

Figure 1. Lambert function for $x \geq-\frac{1}{e}$. There are two branches on the interval $\left[-\frac{1}{e} ; 0\right)$ [29]. The continuous line represents the principal branch $W_{0}$.

solution $Q_{\infty}=0$. The other solution is given by the second value of $W(x)$, that must be read on its principal branch, denoted as $W_{0}$ [29]. It is the non-dotted part of the curve represented in Figure 1. Therefore the unique strictly positive solution $f(x)=x$ is given by:

$$
Q_{\infty}=A+\frac{1}{B} \cdot W_{0}\left(-A B \cdot e^{-A B}\right)
$$

Moreover, $Q_{\infty}$ is an attractive fixed point of $f$ :

$$
\left|f^{\prime}\left(Q_{\infty}\right)\right|=\left|W_{0}\left(-A B \cdot e^{-A B}\right)\right|<1
$$

since

$$
-A B \cdot e^{-A B} \in\left(-\frac{1}{e} ; 0\right) \text { for } A B>1 .
$$

As, in addition, $f$ is continuously differentiable, this proves that there is linear convergence of the sequence $Q(t)$ to $Q_{\infty}$.

The value of this fixed point belongs to the following interval:

$$
0<A-\frac{1}{B}<Q_{\infty}<A
$$

Good approximations of the values of $W_{0}(x)$ can be computed thanks to its Taylor serie [31], converging for $|x|<\frac{1}{e}$ (d'Alembert ratio test):

$$
W_{0}(x)=\sum_{n=1}^{\infty} \frac{(-n)^{n-1}}{n !} x^{n}
$$




\subsection{A criterion to assess the potential of an archi- tectural model for limit production}

$Q_{\infty}$ is an increasing function of $A$. This is coherent with the interpretation of $A\left(=E_{\infty} \mu S_{p}\right)$ : increasing the environmental variable $E$, the coefficient of biomass conversion efficiency $\mu$ or the maximal ground surface projection of blades naturally results in increasing the limit production.

Using the first term of this development of $W_{0}$, we can see that $Q_{\infty}$ is also an increasing function of $B$. Therefore calculating the value of $B$ for different topological parameters provides a criterion to evaluate the performance of different architectural models.

In application, we give the expression of $B$ for two particular architectural models: Corner and Leeuwenberg models, as represented in Figure 2. The case of the Roux model can be found in [30].

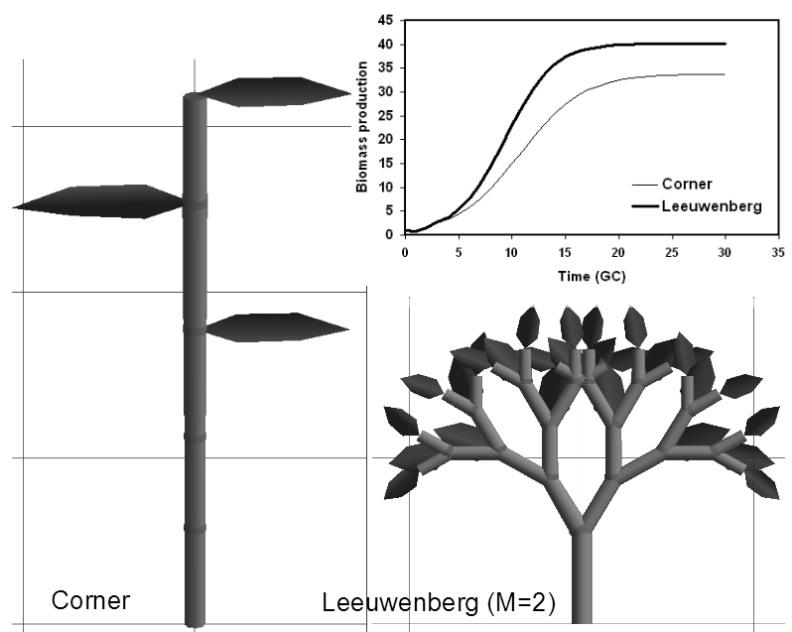

Figure 2. Corner and Leeuwenberg architectural models: comparison of biomass productions. The simulated values are consistent with the numerical results found after solving eq. 3 as shown in fig. 3 .

No fruits are considered and all organs are of the same physiological ages. $P^{a}$ and $P^{i}$ represent respectively blade and internode sink strength while $\phi^{a}$ and $\phi^{i}$ are sink variation functions.

- Corner model: there is one more metamer at each growth cycle.

$$
\begin{aligned}
& \forall t \geq 1, N^{a}(t)=N^{i}(t)=1 \\
& \forall t \geq t_{\text {exp }}, D(t)=\sum_{j=1}^{t_{\text {exp }}} P^{a} \cdot \phi^{a}(j)+\cdot P^{i} \cdot \phi^{i}(j) \\
& B=\frac{k P^{a}}{e S_{p}} \frac{\sum_{n=1}^{t_{a}} \sum_{j=1}^{n} \phi^{a}(j)}{\sum_{j=1}^{t_{\text {exp }}} P^{a} \phi^{a}(j)+P^{i} \phi^{i}(j)}
\end{aligned}
$$

If $t_{a}=1, B$ does not depend on the sink variation coefficients and the condition for a strictly positive limit production $(A B>1)$ can be further simplified to:

$$
\frac{E_{\infty} \mu k}{e} \frac{P^{a}}{P^{a}+P^{i}}>1
$$

A numerical value of the limit production is given in the example of Figure 3.

- Leeuwenberg model: each apical bud gives birth to $M$ new metamers:

$$
\begin{aligned}
& \forall t \geq 1, N^{a}(t)=N^{i}(t)=M^{t-1} \\
& \forall t \geq t_{\text {exp }}, \quad D(t)=\sum_{j=1}^{t_{\text {exp }}} M^{t-j+1}\left(P^{a} \phi^{a}(j)+P^{i} \phi^{i}(j)\right) \\
& B=\frac{k P^{a}}{e S_{p}} \sum_{n=1}^{t_{a}} \sum_{j=1}^{n} \frac{\phi^{a}(j)}{\sum_{j^{\prime}=1}^{t_{\text {exp }}} M^{j-j^{\prime}}\left(P^{a} \phi^{a}\left(j^{\prime}\right)+P^{i} \phi^{i}\left(j^{\prime}\right)\right)}
\end{aligned}
$$

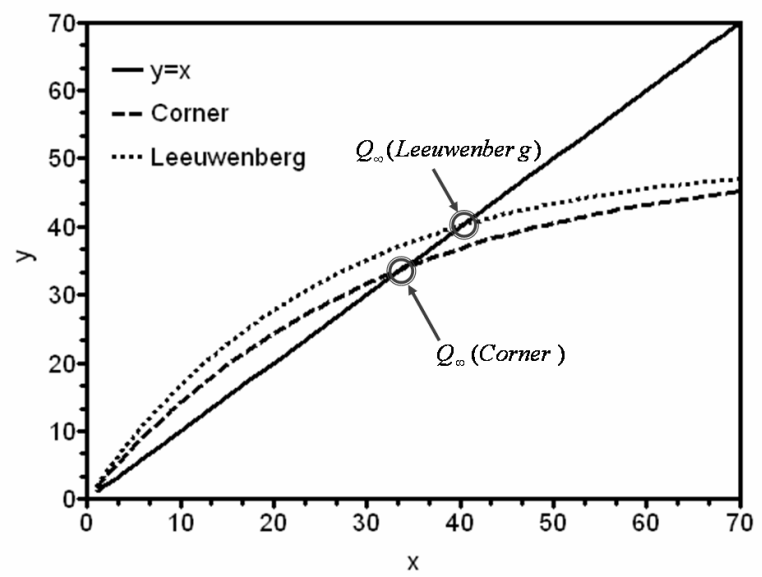

Figure 3. Graphical resolution of Eq.3: $Q_{\infty}=A(1-$ $\left.e^{-B \cdot Q_{\infty}}\right)$ for Corner and Leeuwenberg $(\mathrm{M}=2)$ architectural models. The parameter values are given in table 1. Numerically (eq. 7), the limit productions are: $Q_{\infty \text { Corner }}=33.79$ and $Q_{\infty \text { Leeuwenberg }}=40.16$

Table 1. Parameter values of simulations of Fig. 3.

\begin{tabular}{|c|ccc|}
\hline$t_{a}=3 \quad \begin{array}{c}S_{p}=500 \\
t_{\text {exp }}=3\end{array}$ & $\begin{array}{c}E=1 \\
P^{a}, P^{i}=1\end{array}$ & $\begin{array}{c}k=0.06 \\
\phi^{a}, \phi^{i}=1\end{array}$ & $\begin{array}{l}\mu=0.1 \\
\text { It gives: } A=50\end{array}$ \\
$B_{\text {Corner }}=\frac{k \cdot P^{a}}{e \cdot S_{p}} \frac{2}{P^{a}+P^{i}}=0.033$ \\
$B_{\text {Lee }}=\frac{k P^{a}}{e \cdot S_{p}\left(P^{a}+P^{i}\right)}\left(\frac{3 M^{2}+2 M+1}{M^{2}+M+1}\right)=0.04$
\end{tabular}

In the illustration presented in Figures 2 and 3, the limit production for the Leeuwenberg model is higher than that of the Corner model. In that case $\left(T_{\exp }=3\right)$, it can be shown that as soon as its number of buds per metamer is $M>1$, the 
limit production of a Leeuwenberg model is necessary higher than that of a Corner model. As all functional parameters are chosen identical, this difference is purely related to the architectural model that develops. However, this trend would be likely to change if hydraulic resistances of axes were considered as there are more internodes in the Leeuweberg tree. Note that the seed biomass (initial condition of the system) has no effect neither on the limit production nor on the condition $(A B>1)$ to reach a stable positive value.

\section{Invariances of biomass production}

To analyze the importance of architecture on plant functioning, it is also interesting to study the cases when, contrary to the cases presented above, topological development has no influence on biomass production. In fact, it can be shown that under some conditions, plants with different topologies have the same biomass production at every cycle of their growth (not only the limit production of the steady state).

Hereafter we consider the case when all organs have the same physiological age $\left(P_{m}=1\right)$ and there is no ring compartment in the demand.

\subsection{Case 1: immediate expansion of organs}

Under these assumptions $\left(T_{\exp }=1\right)$, the recurrence equation of biomass production $(\mathrm{Eq} 1)$ is:

$$
Q(t)=E \mu S_{p}\left[1-\exp \left(-\frac{k P^{a}}{e S_{p}} \sum_{i=1}^{t_{a}} N_{t-i+1}^{a} \frac{Q(t-i)}{D(t-i)}\right)\right]
$$

In that case, there is a linear relationship between the numbers of organs and leaves with the proportionality constant $k^{o}$. Thus the demand of the shoot part is the sum of the demand of new organs only (internodes with sink $P^{i}$ and fruits or flowers with sink $P^{f}$ ):

$$
D(t)=\left(P^{a}+k^{i} \cdot P^{i}+k^{f} \cdot P^{f}\right) \cdot N^{a}(t+1)
$$

where $N^{a}(t+1)$ is the number of new leaves at GC $t+1$ (the demand is calculated at end of GC so $D(t)$ account for the new organs appearing at the following GC). As the number of blades $N^{a}(t+1)$ is a factor of this equation, the ratio in equation 8 simplifies. Therefore the recurrent equation giving the biomass production at $\mathrm{GC} t$, Equation (1), can be written as :

$$
Q(t)=E \mu S_{p}\left[1-\exp \left(-\frac{k}{e S_{p}} \frac{P^{a} \cdot \sum_{i=1}^{t_{a}} Q(t-i)}{P^{a}+k^{i} P^{i}+k^{f} P^{f}}\right)\right]
$$

Thus, if the initial condition (biomass of the seed $Q_{0}$ ) is fixed, the sequence $\left(Q_{t}\right)_{t \in \mathbb{N}}$ is fixed. It means that two plants verifying these assumptions and having leaves with the same functioning duration produce exactly the same

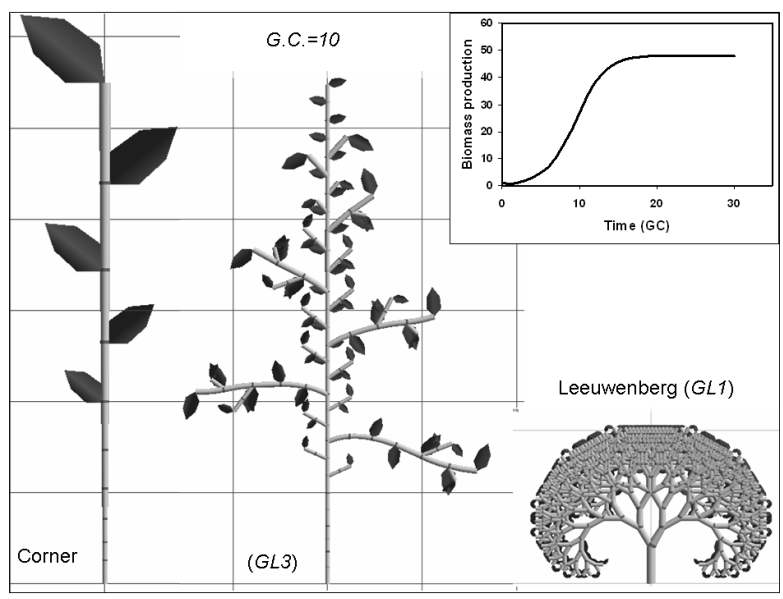

Figure 4. Simulation of plant growth with different topologies but same biomass production (Case 1: $\left.t_{\text {exp }}=1, P_{m}=1, D_{\text {ring }}=0\right)$. The functional parameters are: $t_{a}=5, P^{a}=1, P^{i}=1.5, \mu=0.1, e=0.06$, $S_{p}=500$.

biomass amount at each GC, whatever their topologies are. Figure 4 shows some simulation illustrations with Corner and Leeuwenberg models $(G L 1)$ and with a plant simulated with the $G L 3$ version. The same arguments remain valid if there are several physiological ages in the plant $\left(P_{m}>1\right)$ but with a constant proportionality between sinks of organs of each PA, i.e. under the following condition:

$$
\exists k^{o}, \forall p \in 1 \ldots P_{m}, P_{p}^{o} \cdot N_{p}^{o}(t)=k^{o} \cdot P_{p}^{a} \cdot N_{p}^{a}(t)
$$

The same calculations apply also for the case (purely theoretical) where there is immediate expansion $\left(T_{\exp }=1\right)$ of all organs with only one physiological age $\left(P_{m}=1\right)$ and ring demand depending on the number of leaves with a constant number of leaves all along its life.

\subsection{Case 2: expansion duration of several G.C. $\left(t_{\exp } \geq 1\right)$ with same shape of sink variation function for all organs}

Again, the number of internodes is proportional to the number of leaves so we can write:

$$
D(t)=\left(P^{a}+k^{i} \cdot P^{i}\right) \sum_{i=1}^{M i n\left(t, t_{e x p}\right)} N^{a}(t-i+1) \phi^{a}(i)
$$

Intuitively, it would seem possible to simplify the recurrent equation for the biomass production, as the same sink variation coefficients and numbers of leaves appear in the ratio of the exponential factor. In fact it is not always true (and this can be checked from the examples of Figure 2). More precisely, after several rearrangements, the above 
equation can be written under the form:

$$
\begin{aligned}
& Q_{t}=E \mu S_{p}\left[1-\exp \left(\frac{-k P^{a}}{e S_{p}\left(P^{a}+k^{i} P^{i}\right)} \cdot C\right)\right] \\
& \text { with } C=\sum_{i=1}^{t_{a}} \frac{Q_{n-i} \sum_{j=1}^{t_{a}-i+1} N^{a}(t-i-j+2) \phi^{a}(j)}{\sum_{j=1}^{t_{e x p}} N^{a}(t-i-j+2) \phi^{a}(j)}
\end{aligned}
$$

One can see that the ratio does not simplify as the number of terms in the sums are different. The residual terms are due to the fact that when a leaf dies, its whole biomass ceases activity regardless of the time it was allocated to the leaf.

3.2.1. Residual term due to leaf senescence. An alternative possibility would be to consider progressive senescence of leaf. The amount of leaf biomass allocated at GC $t$ would cease activity at GC $t+t_{a}$. In that case, equation 10 simplifies to:

$$
Q(t)=E \mu S_{p}\left[1-\exp \left(\frac{-k P^{a} \sum_{i=1}^{t_{a}} Q(t-i)}{e S_{p}\left(P^{a}+k^{i} P^{i}\right)}\right)\right]
$$

Current modelling choice in GreenLab:

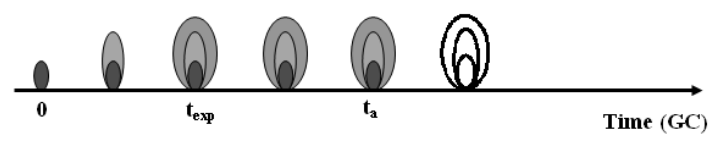

Version without residuals: progressive senescence

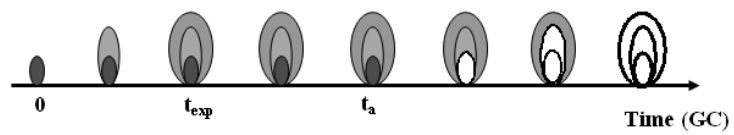

Figure 5. Leaf senescence (in white): in the current version of GreenLab, leaf activity ceases abruptly: it generates a residual term in the recurrent equation of biomass production. An alternative choice would be to consider that leaf senescence is progressive: every unit of leaf biomass would remain active during $t_{a}$ growth cycles.

Thus with this modelling of leaf senescence, it is possible to find plants with different topological development but similar sequences of biomass production. Note that Equation 11is the same than Equation 9: expansion durations and sink variation shapes have no influence on the sequences of biomass production.

3.2.2. Case of infinite functioning duration of leaves. Apart from this question, the simplification can also be done when the functioning duration of leaves $\left(t_{a}\right)$ is infinite. Indeed in this case, leaves never become senescent. Figure
6 presents some example of simulated plant growth with the same biomass production and allocation to leaf and internode compartments.

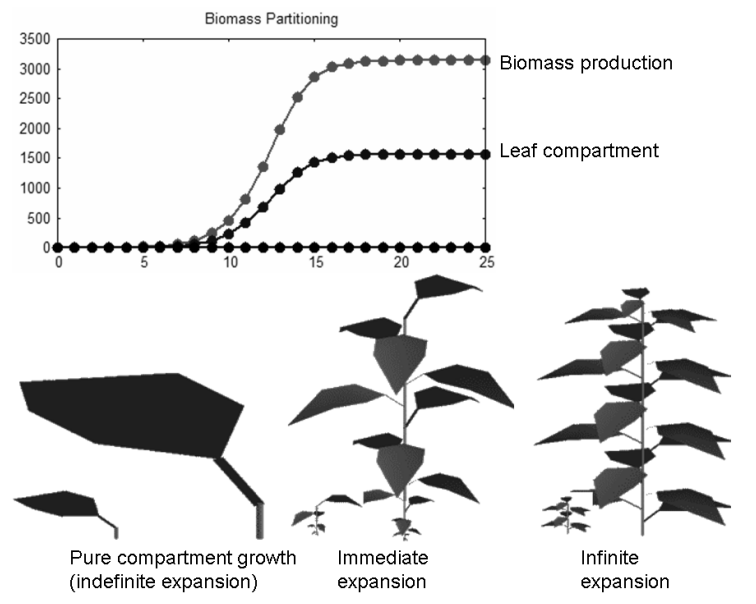

Figure 6. Examples of simulated plants with same biomass production and allocation to blade and internode compartments. Leaf functioning time is infinite. Expansion is immediate or infinite. For each plant, all organ kinds have the same shape of sink variation.

To conclude, similar production sequences can be found for plants with different topological developments as soon as they respect one of the following conditions:

- Immediate expansion $\left(t_{\exp }=1\right)$, one physiological age $\left(P_{m}=1\right)$, no rings (or ring demand proportional to the number of leaves and a constant number of leaves)

- Expansion duration for several cycles $\left(t_{e x p} \geq 1\right)$, one physiological age $\left(P_{m}=1\right)$ and same sink variation shape for all organs, provided that one of the following conditions is verified:

- Leaf functioning duration is infinite (at least equal to the plant chronological age)

- Leaf senescence is progressive: every unit of leaf biomass remains active during exactly $t_{a}$ growth cycles.

In other cases, there is no simplification of the ratio of number of leaves to the total demand and therefore the topological development of the plant influences its production.

\section{Discussion and conclusion}

This paper presents a limit state and behaviour analysis of the GreenLab model. It has highlighted the interest of models to assess the performances of different topological developments to maximize the plant potential production. An index defining the plant potential performance was presented and would allow comparing different strategies of development. It seems logical that the number of organs appearing at 
each cycle has an effect on plant biomass production during the establishment phase. However, once biomass production stabilizes, the increase of blade surface has no impact on the production. Therefore it was not clear whether the influence of architecture remained important. This question is particularly crucial for process-based models where only compartments of biomass are usually considered [32]-[35]. The results of our analysis show that in most cases, the number of organs is determinant for the value of limit biomass production. The conditions under which it is possible to get similar sequences of biomass productions with different topological developments revealed themselves to be mainly theoretical and are hardly encountered in nature. Indeed, as soon as the root compartment is considered, the simplifications of the equations presented above no longer hold. Moreover, similar study should be done including the underground part: for instance, Fitter et al [36] analysed the influence of root architecture for soil exploitation efficiency using simulations. However, using sensitivity analysis to further study the distance between different plants as far as their potential limit production is concerned would bring more detailed insights.

The limit state analysis can provide clues to discriminate between several modelling choices. For instance in [26], the effect of the calculation mode for ring demand on limit biomass production in GreenLab was studied. It was shown that one of the expressions proposed (ring demand proportional to total biomass production) induces lower limit production values than the two other ones (ring demand proportional to the number of leaves or ring demand proportional to the ratio of biomass supply to demand). By comparison with the qualitative biological knowledge, these properties can be criteria to guide modeller's choice.

Studying invariants in a model can be interesting before beginning the procedure of parametric identification. Here we showed that several architectures can produce the same trajectory of biomass production. Although the conditions found to get invariant biomass production imply that these cases are purely theoretical, it is likely that it exists real cases when the difference engendered by two different sequences of topological development is low. Therefore if the only data available is biomass, there might be several appropriate solutions when one aims at estimating the parameters driving the topological development [37]. This case could be encountered if data collected at the level required for processbased models (i.e. at compartment level) were used to fit a functional-structural model. In that case, more a priori knowledge should be incorporated: for instance, a default topology can be defined based on the botanical study of the corresponding species. Then parameters can be fitted to drive the variations of this potential topology according to the plant internal state of trophic competition and in relation with environmental constraints.

\section{Acknowledgment}

The authors thank V. Le Chevalier for his corrections on this manuscript.

\section{References}

[1] T. Fourcaud, X. Zhang, A. Stokes, H. Lambers, and C. Korner, "Plant growth modelling and applications: the increasing importance of plant architecture in growth models," Annals of Botany, vol. 101, pp. 1053-1063, 2008.

[2] R. Sievänen, E. Nikinmaa, P. Nygren, H. Ozier-Lafontaine, J. Perttunen, and H. Hakula, "Components of a functionalstructural tree model," Annals of Forest Sciences, vol. 57, pp. 399-412, 2000.

[3] P. Prusinkiewicz, "Modeling plant growth and development," Current opinion in plant biology, vol. 7, no. 1, pp. 79-84, 2004.

[4] J. Perttunen, R. Sievänen, E. Nikinmaa, H. Salminen, H. Saarenmaa, and J. Väkevä, "LIGNUM: a tree model based on simple structural units," Annals of Botany, vol. 77, pp. 8798, 1996.

[5] C. Godin and Y. Caraglio, "A multiscale model of plant topological structures," Journal of Theoretical Biology, vol. 191, pp. 1-46, 1998.

[6] W. Kurth, "Specifications of morphological models with Lsystems and relational growth grammars," Journal of Interdisciplinary Image Science, vol. 5, 2007.

[7] G. Lopez, R. Favreau, C. Smith, E. Costes, P. Prusinkiewicz, and T. DeJong, "Integrating simulation of architectural development and source-sink behaviour of peach trees by incorporating Markov chains and physiological organ function submodels into L-PEACH," Functional Plant Biology, vol. 35, pp. 761-771, 2008.

[8] E. Costes, C. Smith, M. Renton, Y. Guédon, P. Prusinkiewicz, and C. Godin, "MApple-T: simulation of apple tree development using mixed stochastic and biomechanical models," Functional Plant Biology, vol. 35, pp. 936-950, 2008.

[9] J. Perttunen, R. Sievänen, and E. Nikinmaa, "LIGNUM: a model combining the structure and the functioning of trees," Ecological Modelling, vol. 108, pp. 189-198, 1998.

[10] B. Zheng, L. Shi, Y. Ma, Q. Deng, B. Li, and Y. Guo, "Comparison of architecture among different culitvars of hybrid rice using a spatial light model based on 3-D digitising," Functional Plant Biology, vol. 35, pp. 900-910, 2008.

[11] M. Cieslak, C. Lemieux, J. Hanan, and P. Prusinkiewicz, "Quasi-monte carlo simulation of the light environment of plants," Functional Plant Biology, vol. 35, pp. 837-849, 2008.

[12] C. Robert, C. Fournier, B. Andrieu, and B. Ney, "Coupling a 3D virtual wheat plant model with a Septoria tritici epidemic model (Septo3D): a new approach to investigate plant-pathogen interactions linked to canopy architecture," Functional Plant Biology, vol. 35, pp. 997-1013, 2008. 
[13] H. Yan, M. Kang, P. De Reffye, and M. Dingkuhn, "A dynamic, architectural plant model simulating resourcedependent growth," Annals of Botany, vol. 93, pp. 591-602, 2004.

[14] P. Cournède, M. Kang, A. Mathieu, J. Barczi, H. Yan, B. Hu, and P. de Reffye, "Structural factorization of plants to compute their functional and architectural growth," Simulation, vol. 82, no. 7, pp. 427-438, 2006.

[15] P. Prusinkiewicz, "Modeling of spatial structure and development of plants : a review," Scientia Horticulturae, vol. 74, pp. 113-149, 1998.

[16] M. Kang, P.-H. Cournède, J.-P. Quadrat, and P. de Reffye, "A stochastic language for plant topology," in Plant Growth Models and Applications (PMA06, Beijing, China), T. Fourcaud and Z. X.P., Eds. IEEE (Los Alamos, California), 2006.

[17] C. Loi and P.-H. Cournède, "Generating functions of stochastic L-systems and application to models of plant development," Discrete Mathematics and Theoretical Computer Science Proceedings, vol. AI, pp. 325-338, 2008.

[18] W. Kurth, "Some new formalisms for modelling the interactions between plant architecture, competition and carbon allocation," in 4th workshop on individual-based structural and functional models in ecology. Wallenfels, Bayreuther forum ökologie, 91996.

[19] D. Barthélémy, Y. Caraglio, and E. Costes, "Architecture, gradients morphogénétiques et âge physiologique chez les végétaux," in Modélisation et simulation de l'architecture des végétaux, ser. Sciences Update, J. Bouchon, Ed. INRA, 1997, pp. 89-136.

[20] Y. Zhang, D. Reed, P. Cattelino, M. Gale, E. Jones, H. Liechty, and G. Mroz, "A process-based growth model for young red pine," Forest Ecology and Management, vol. 69, pp. 21-40, 1994.

[21] P. Prusinkiewicz and A. Lindenmayer, The Algorithmic Beauty of Plants. Springer-Verlag, New-York, 1990.

[22] M. Kang, P. de Reffye, J. Barczi, B. Hu, and F. Houllier, "Stochastic 3D tree simulation using substructure instancing," in Plant Growth Models and Applications PMA03, B. Hu and M. Jaeger, Eds. Tsinghua University Press and Springer (Beijing, China), 2003, pp. 154-168.

[23] A. Mathieu, P.-H. Cournede, V. Letort, D. Barthelemy, and P. de Reffye, "A dynamic model of plant growth with interactions between development and functional mechanisms to study plant structural plasticity related to trophic competition," Annals of Botany, vol. In Press, p. mcp054v1, 32009.

[24] P. de Reffye, M. Goursat, J. Quadrat, and B. Hu, "The dynamic equations of the tree morphogenesis GreenLab model," in Plant Growth Models and Applications, B. Hu and M. Jaeger, Eds. Tsinghua University Press and Springer, Beijing, China, 2003, pp. 108-117.

[25] Y. Guo, Y. Ma, Z. Zhan, B. Li, M. Dingkuhn, D. Luquet, and P. de Reffye, "Parameter optimization and field validation of the functional-structural model GreenLab for maize," Annals of Botany, vol. 97, pp. 217-230, 2006.
[26] V. Letort, "Adaptation of the greenlab growth model to plants with complex architectures and multi-scale analysis of sourcesink relationships for parametric," Ph.D. dissertation, Ecole Centrale Paris, 2008.

[27] D. Chauveheid, "Formalisation et étude d'un système complexe dans la théorie des systèmes dynamiques," Master's thesis, Ecole Centrale Paris, France, 2008, rapport de projet scientifique.

[28] A. Mathieu, P. Cournède, D. Barthélémy, and P. de Reffye, "Rhythms and alternating patterns in plants as emergent properties of a model of interaction between development and functioning," Annals of Botany, vol. 101, no. 8, pp. 1233 $1242,2008$.

[29] R. M. Corless, G. H. Gonnet, D. E. G. Hare, D. J. Jeffrey, and D. E. Knuth, "On the Lambert W function," Advances in Computational Mathematics, vol. 5, pp. 329-359, 1996.

[30] V. Grange, "GreenLab mathematical model behavior analysis on Roux architectural model," 2006, rapport de stage d'option scientifique, LIAMA.

[31] R. M. Corless and D. E. Jeffrey, D. J.and Knuth, "A sequence of series for the Lambert W function," in International Symposium on Symbolic and Algebraic Computation, Maui, Hawaii. New York: ACM Press, 1997, pp. 197-204.

[32] R. Sievänen and T. Burk, "Fitting process-based models with stand growth data: problems and experiences," Forest Ecology and Management, vol. 69, no. 1-3, pp. 145-156, 1994.

[33] H. Bossel, "Treedyn3 forest simulation model," Ecological Modelling, vol. 90, no. 3, pp. 187 - 227, 1996. [Online]. Available: http://www.sciencedirect.com/science/article/B6VBS3VWK7V1-6/2/85fd01f297515994ed20a4ab40408879

[34] A. Mäkelä, "A carbon balance model of growth and selfpruning in trees based on structural relationships." Forest Science, vol. 43, no. 1, pp. 7-24, 1997.

[35] C. R. Schwalm and A. R. Ek, "A process-based model of forest ecosystems driven by meteorology," Ecological Modelling, vol. 179, no. 3, pp. 317 - 348, 2004. [Online]. Available: http://www.sciencedirect.com/science/article/B6VBS4CY0GMM-1/2/681dfd673f0f9a0df5c4243258126f61

[36] A. H. Ftter, T. Stickland, M. L. Harvey, and G. Wilson, "Architectural analysis of plant root systems 1. architectural correlates of exploitation efficiency," New Phytologist, vol. 118, pp. 375-382, 1991.

[37] V. Letort, P.-H. Cournède, A. Mathieu, P. de Reffye, and T. Constant, "Parameter identification of a functionalstructural tree growth model and application to beech trees (Fagus sylvatica)," Functional Plant Biology, vol. 35, pp. 951-963, 2008. 